\title{
Analysis of the Curative Effect of the Treatment of the Three-dimensional Conformal Radiotherapy Combination with the Induction Chemotherapy in the Middle-late Nasopharyngeal Carcinoma
}

\author{
Min $\mathrm{Ma}^{1, \mathrm{a}}$, Hongying $\mathrm{Lin}^{1,}{ }^{1}$, Shengcun Zhang ${ }^{1}$ \\ ${ }^{1}$ Department of Cancer and Hematology, The third People's Hospital of Jinan, Jinan 250132, \\ China \\ amamin@126.com \\ *Corresponding author linlin88@163.com
}

\begin{abstract}
Keywords: Three dimensional conformal radiotherapy; Induction chemotherapy; Locally advanced nasopharyngeal carcinoma
\end{abstract}

\begin{abstract}
Objective: To observe and explore the clinical curative effect of the three dimensional conformal radiotherapy combination with the induction chemotherapy to treat the middle-late nasopharyngeal carcinoma patients. Methods: We select from February 2012 to February 2014 the clinical data of 98 middle-late nasopharyngeal carcinoma patients. They were retrospectively analyzed, and were divided into observation group and control group according to the treatment, each group are 49 cases, the observation group are received three dimensional conformal radiotherapy combination with the induction chemotherapy, the control group are accepted the three-dimensional conformal radiotherapy. Three dimensional conformal radiation therapies, chemotherapy is to TPF scheme, to observe the indexes including nasopharyngeal tumour and neck swelling lymph nodes complete remission rates, 1 year and 3 years of progression-free survival (PFS) and overall survival (OS), the incidence of adverse reactions. Results: Two groups of nasopharyngeal tumours and neck swelling lymph nodes complete remission rate, there was statistically significant difference comparison $(\mathrm{P}<0.05)$, the observation group of patients 1 year and 3 years of PFS and OS is significantly higher than the control group, significant differences are statistically significant $(\mathrm{P}<0.05)$. Conclusion: Three dimensional conformal radiotherapy combination with the induction chemotherapy can improve the PFS and OS of the middle-late stage nasopharyngeal carcinoma patients, although the incidence of adverse reactions, it does not affect the completion of treatment, and it is worthy of clinical popularization and application.
\end{abstract}

\section{Introduction}

The nasopharyngeal carcinoma is a common malignant tumour, early patients with radiation therapy effect is remarkable, but most of patients with nasopharyngeal carcinoma in China see a doctor when $\mathrm{f}$ is clinical period, local recurrence and distant metastasis after radiotherapy alone become a thorny issue. Nasopharyngeal carcinoma is the most common head and neck malignant tumour, radiation treatment as the main treatment method, due to the anatomical characteristics of nasopharyngeal and tumour growth characteristics, middle-late nasopharyngeal carcinoma in patients with both the occupy high proportion. The same period and at the same time, radiation and chemotherapy treatment for locally advanced nasopharyngeal carcinoma has become the consensus of experts, but at the same time of radiation and chemotherapy for acute reaction is often more severe, and the intensity modulated conformal radiation therapy while guarantee the target dose, better protect normal tissue, reduce radiation reaction. This article selects from February 2012 to February 2014, the clinical data of 98 patients with nasopharyngeal carcinoma (NPC) middle-late were retrospectively analyzed; now the curative effect of statistics are as follows. 


\section{Materials and Methods}

\section{Materials}

We select from February 2012 to February 2014, the clinical data of 98 patients with nasopharyngeal carcinoma (NPC) middle-late were retrospectively analyzed, according to the treatment were divided into observation group and control group all the 49 cases, all patients were in line with the middle-late criteria for the diagnosis of nasopharyngeal carcinoma (NPC). The observation group, 32 cases were male, female were 17 cases, aged $43 \sim 78$, the average age (65.7-10.6). The control group 33 cases were the male, 16 cases were the female, age $43 \sim 78$ years old, average age (65.7-10.6). Two groups of patients in such aspects as age, sex, disease differences had no statistical significance $(\mathrm{P}>0.05)$, comparable.

\section{The inclusion criteria}

(1) The score of Karnofsky is 80 points or more; (2) The CT scans have measurable lesions; (3) The routine blood and liver and kidney function without exception; (4) The abdominal B to exceed, chest CT, bone ECT distant organ metastasis.

\section{Methods}

The observation group received three dimensional conformal radiotherapy in combination with the induction chemotherapy, the control group accepted the three dimensional conformal radiotherapy. Three-dimensional conformal radiotherapy, the use of linear accelerator $6 \mathrm{MV}-\mathrm{X}$-ray, routine dose divided, according to the $200 \mathrm{cGy} /$ (time-d), five times a week, the seven to eight weeks in a row. Tumor target to imaging data show the range for irradiation range. Clinical target is based on the macroscopic tumor area, greater risk tumor infiltration of outside the area to put $1 \mathrm{~cm}$; Other areas outside the $5 \mathrm{~mm}$. Planning target is based on the clinical target area, then put 2-5 $\mathrm{mm}$. By using 4-6 coplanar wild or non coplanar wild, with $95 \%$ isodose line coverage plans to target. Important tissues and organs, such as the pituitary gland, brain stem and spinal cord to give safe dose range. Planning target is of 27.9-71.2 $\mathrm{cm}^{3}$ volume, the median size of $42.5 \mathrm{~cm}^{3}$. The exposure dose for 60-75 Gy, median dose was 66 Gy. Chemotherapy regimens: since the radiation 1 day to give TPF scheme chemotherapy, $21 \mathrm{~d}$ to 1 cycle, stay 4 cycles: he Isaiah $60 \mathrm{mg} / \mathrm{m}^{2} \mathrm{dl}$ Dorsey, cisplatin, $30 \mathrm{mg} / \mathrm{m}^{2} \mathrm{dl}-3,5$ - fluorouracil $500 \mathrm{mg} / \mathrm{m} 2 \mathrm{dl}-5$ intravenous drip; Chemotherapy at the same time gives prevention and treatment of adverse drug reactions associated with radiation and chemotherapy treatment. Control group accept simple three-dimensional conformal radiotherapy, scheme is the same in the observation group. Observation indexes including nasopharyngeal tumor and neck swelling lymph nodes complete remission rates, 1 year and 3 years of progression-free survival (PFS) and overall survival (OS), the incidence of adverse reactions.

\section{Statistical processing}

With mathematical statistics software SPSS19.0 for data collection and statistical analysis, and chi-square test, $\mathrm{P}<0.05$, significant difference has statistical significance.

\section{Results}

Two groups of nasopharyngeal tumours and neck swelling lymph nodes complete remission rate, there was statistically significant difference comparison $(\mathrm{P}<0.05)$, the observation group of patients 1 year and 3 years of PFS and OS is significantly higher than the control group, significant differences are statistically significant $(\mathrm{P}<0.05)$. 
Table 1: The near future curative effect comparison between two groups of middle-late nasopharyngeal carcinoma patients

\begin{tabular}{|c|c|c|c|c|c|c|c|}
\hline \multirow{2}{*}{ Group } & \multirow[b]{2}{*}{$\mathrm{n}$} & \multicolumn{3}{|c|}{ Nasopharyngeal lesions } & \multicolumn{3}{|c|}{ The neck metastasis lymph nodes } \\
\hline & & $\mathrm{CR}$ & PR & $\mathrm{CR}+\mathrm{PR}$ & CR & PR & $\mathrm{CR}+\mathrm{PR}$ \\
\hline $\begin{array}{l}\text { The } \\
\text { observation } \\
\text { group }\end{array}$ & 49 & $\begin{array}{c}45 \\
(91.84)\end{array}$ & $4(8.16)$ & 100 & $\begin{array}{c}43 \\
(87.76)\end{array}$ & $6(12.24)$ & 100 \\
\hline $\begin{array}{l}\text { The control } \\
\text { group }\end{array}$ & 49 & $\begin{array}{c}31 \\
(63.27)\end{array}$ & $\begin{array}{c}15 \\
(30.61)\end{array}$ & 93.88 & $\begin{array}{c}29 \\
(59.18)\end{array}$ & $\begin{array}{c}16 \\
(32.66)\end{array}$ & 91.84 \\
\hline $\begin{array}{c}\chi^{2} \\
\text { P value }\end{array}$ & & & & $\begin{array}{l}6.736 \\
<0.05\end{array}$ & & & $\begin{array}{c}8.9324 \\
<0.05\end{array}$ \\
\hline
\end{tabular}

\section{Discussion}

The local recurrence and distant metastasis of nasopharyngeal carcinoma (NPC) middle-late rate is as high as $40 \% \sim 50 \%$. Using traditional external exposure technology, due to open mouth, bite difficulties and parotid gland injury, inflammation of the oral mucosa and bone marrow suppression adverse reactions such as difficult to further improve the target dose, and the nasopharyngeal carcinoma intensity modulated conformal radiation therapy, can greatly improve the radiation gain ratio, to the greatest extent to concentrate radiation dose in target areas, and further reduce the surrounding normal tissue damage, so as to improve the local control and reduce the adverse reaction of tumor. Nasopharyngeal bit depth, surrounded by blood vessels, nerves surgical treatment is extremely limited. The pathology of nasopharyngeal carcinoma (NPC) has mostly low differentiated squamous carcinoma (90\%), sensitivity to radiotherapy, so is the preferred treatment of nasopharyngeal carcinoma radiotherapy. Early nasopharyngeal carcinoma radiotherapy can obtain satisfactory results, but the middle-late (III, IV) of nasopharyngeal carcinoma radiotherapy curative effect is unsatisfactory, the main cause of treatment failure is local control and distant metastasis, so many scholars focus to put on, combined with the comprehensive treatment way. Induction chemotherapy is also called the neoadjuvant chemotherapy before radiotherapy, its advantage lies in: (1) no fibrosis caused by radiation therapy, tumor blood supply is good, is advantageous to the chemotherapy drug in nasopharyngeal carcinoma distribution and utilization of primary tumors and lymph node metastasis lesions, tumor chemotherapy to reduce load, improve blood supply, thus reducing tumor anaerobic cells, increase tumor susceptibility to radiation therapy, to some extent improve the tumor local control, killing sub clinical lesions, thus improve the curative effect; Nutritional status is good, (2) patients before radiotherapy can increase patient's sensitivity to chemotherapy and tolerance; (3) the systemic chemotherapy can effectively kill the viscera of subclinical metastatic lesions. (4) The direct effect of induction chemotherapy for nasopharyngeal carcinoma (NPC) is a short period of time; make the local tumor and regional lymph node cancer, serious stuff such as blood, symptoms such as headache, nasal congestion eased significantly. DDP is cell cycle non-specific chemotherapy drugs, it is one of the most effective drugs for the treatment of nasopharyngeal carcinoma, regular doses of bone marrow suppression is lighter, by inhibiting the sub lethal damage repair, oxygenation and cycle redistribution can strengthen the effect of radiation to kill cancer. Is given priority to with DDP chemotherapy scheme is recognized as good, head and neck squamous cell carcinomas and 5 - Fu have synergistic anticancer, CF can increase the anti-cancer effect of 5 - Fu. Mask fixed light USES the same position, place a good repeatability. Face, neck, joint wild avoided and neck doses of "hot spots". After the process of CT positioning, target TPS sketch, optimization of dose curve, on the edge of the conformal radiotherapy of tumor dose steep fall, better protect the adjacent normal tissues, such as the brain stem, spinal cord, important organs such as eye, increase the tumor dose and improve patient quality of life.

Due to the early symptoms of non-specific nasopharyngeal carcinoma (NPC) and the biological characteristics of nasopharyngeal carcinoma (NPC), 70\% of patients have been found for the period. 
Radiation therapy is recognized at home and abroad at present application in the treatment of choice for nasopharyngeal carcinoma (NPC), the 5-year survival rate is $34 \%$ - $34 \%$ of early nasopharyngeal carcinoma, and advanced the 5-year survival rate of nasopharyngeal carcinoma (NPC) is only $20 \%$. In recent years, the application of comprehensive therapy in patients with advanced nasopharyngeal carcinoma in more and more attention, many scholars by radiotherapy in combination with chem otherapy to improve the bureau to control and decrease distant metastasis of nasopharyngeal carcinoma (NPC) rate, further improve the survival rate of patients with nasopharyngeal carcinoma (NPC). Existing literature reported by induction chemotherapy can decrease the rate of distant metastases, to improve the local control and disease-free survival also to have certain effect, but overall survival has not been improved; but in combination with the radiotherapy can increase overall survival. So the radiation and chemotherapy combination therapy for nasopharyngeal carcinoma (NPC) middle-late currently accepted treatment.

As a systemic chemotherapy treatments, in the comprehensive treatment of tumor, plays an important role, especially for poor local treatment, radiotherapy, surgery) cases, application of systemic chemotherapy to kill small metastases, hidden in the body to the primary lesion and regional lymph node metastases are all in good killing effect and prompted to tumor shrinkage and cell synchronization at the same time, enhance the sensitivity of radiotherapy, and the radiation effect of mutual coordination. Most is a poorly differentiated squamous carcinoma nasopharyngeal carcinoma (NPC), the vast majority of all platinum based chemotherapy combined regimen, and the most commonly used chemotherapy at home and abroad is DDP and 5 - Fu. Treat middle-late nasopharyngeal carcinoma using the same radiation and chemotherapy technology has formed a consensus, but the side effects of chemotherapy drugs can increase the radiation response, basically have dry mouth and oral mucosa reaction (burning sensation and pain), etc., can appear after the irradiation, dry mouth, hearing decreased visual acuity, subcutaneous tissue fibrosis, and gastrointestinal reactions such as nausea, vomiting, diarrhea and fatigue, bone marrow suppression, etc.

Roeder scholars reported early nasopharyngeal carcinoma radical radiotherapy of the failure rate was $32.3 \%$ (71/220), local recurrence rate was $51.4 \%$ (43/220), and the distant metastasis rate was $44.5 \%$ (98/220). A large number of clinical observations proved long curative effect in nasopharyngeal carcinoma lines of radiotherapy alone. In recent years, the joint treatment of nasopharyngeal carcinoma (NPC) radiation and chemotherapy for clinical attention, the application of the chemotherapy ways mainly have before radiotherapy after induction chemotherapy, radiation therapy, adjuvant chemotherapy and chemo radiation, of which the most respected chemo radiation. The sarisahin scholars think through a lot of clinical data analysis, such as chemo radiation is the most reasonable treatment period in patients with nasopharyngeal carcinoma. The chemo radiation is the theoretical basis of the scholars thinks those applications such as: (1) chemical drugs can kill tumor cells directly; (2) the chemical can interfere with tumor cell damage repair; (3) chemical has the tumor cells of synchronization, indirectly increased the sensitivity of radiotherapy.

Nasopharyngeal carcinoma in otolaryngology belongs to one of the more common tumors, because its pathogenesis has concealment, when clear diagnosis, more than $75 \%$ of patients develop to middle-late nasopharyngeal carcinoma, radiotherapy is relatively common in clinical treatment of nasopharyngeal carcinoma (NPC) middle-late measures, for the first time after radiotherapy in patients with high local recurrence rate, usually reached $10.2 \% \sim 15.7 \%$, again be radiotherapy 5 -year survival rate was only $23.0 \%$.

Cisplatin is an antineoplastic chemotherapy drug, its role is in the cell cycle is nonspecific, often used in the process of nasopharyngeal carcinoma chemotherapy drugs, ordinary dose can produce mild inhibition of bone marrow, via inhibition damage can be repaired, the oxygenation and periodic redistribution can improve radiation to kill cancer. DDP induced chemotherapy plus 5-FU combined radiation and chemotherapy treatment period nasopharyngeal carcinoma during this period, the effect is more obvious. Chemotherapy drugs can reduce the phenomenon in patients with distant metastasis, tumor cells can be avoided by chemotherapy drugs exposure, radiation therapy 
after the fatal damage repair. Chemotherapy drugs on tumor cell has direct destruction effect, further complete the destroy tumor.

The radiotherapy of nasopharyngeal carcinoma (NPC) how many, now more praise highly is three-dimensional conformal radiotherapy, its good conformal target, through the coplanar and non coplanar more wild illuminate, the exposure dose concentration in the target area, the surrounding normal tissues are not affected by or less by light, it has the incomparable advantages of conventional radiotherapy chemoradiation commonly used chemotherapy scheme is PF, platinum and synergistic anticancer effects of 5 - Fu, this scheme has been the gold standard for chemoradiation scheme selection of nasopharyngeal carcinoma (NPC). Clinical studies have found that many west he match can destroy tumor cells mitosis, have certain curative effect for head and neck cancer, so this research adopts three dimensional conformal radiotherapy synchronous TPF scheme chemotherapy treatment of locally advanced nasopharyngeal carcinoma, the accepted radiotherapy alone were retrospectively analyzed and the clinical data of the middle-late nasopharyngeal carcinoma treated with concurrent chemo radiotherapy, the result shows that: (1) 1 months, six months, 1 year after treatment group only with the radiotherapy of nasopharyngeal tumor complete remission rate and neck swelling lymph nodes complete remission rate is not significant, the reason is that the three dimensional conformal radiotherapy for locally advanced nasopharyngeal carcinoma the reduction effect of local lesion, chemotherapy role has been cut to the shelter. (2) the observation group 1 year and 3 years of PFS and US significantly higher than that of simple radiotherapy group, showed that chemoradiation scheme can improve the middle-late in patients with nasopharyngeal carcinoma (NPC), which are in conformity with the majority of clinical research at home and abroad. (3) The observation group had a significantly higher incidence of bone marrow suppression and gastrointestinal reaction simple radiotherapy group; Incidence of adverse reactions and other simple radiotherapy group has no statistical significance. Adverse reaction is the main problem, chemoradiation is combined treatment result in the inhibition of bone marrow, gastrointestinal tract reaction, the degree of adverse reactions such as renal toxicity, may affect the dose of radiation and chemotherapy.

\section{Conclusion}

Three dimensional conformal radiotherapy combination with the induction chemotherapy can improve the PFS and OS of the middle-late stage nasopharyngeal carcinoma patients, although the incidence of adverse reactions, it does not affect the completion of treatment. The selected three dimensional conformal radiotherapy combination with the induction chemotherapy patients were fully accepted the reservation of radiation and chemotherapy scheme, analysis of the causes and treatment of serious functional status of strictly before scoring, eliminate taboo, effective prevention and treatment on adverse reactions, and it is worthy of clinical popularization and application.

\section{Acknowledgement}

This research was financially supported by the National Science Foundation.

\section{References}

[1] Xueming Sun, Shengfa Su, Chunyan Chen, Fei Han, Chong Zhao, Weiwei Xiao,Xiaowu Deng, Shaomin Huang, Chengguang Lin, Taixiang Lu. Long-term outcomes of intensity-modulated radiotherapy for 868 patients with nasopharyngeal carcinoma: An analysis of survival and treatment toxicities[J]. Radiotherapy and Oncology. 2013(8)

[2] Ya Hua Zhong, Jing Dai, Xiao Yong Wang, Cong Hua Xie, Gang Chen, Lei Zeng, Yun Feng Zhou. Phase II trial of neoadjuvant docetaxel and cisplatin followed by intensity-modulated radiotherapy with concurrent cisplatin in locally advanced nasopharyngeal carcinoma[J]. Cancer Chemotherapy and Pharmacology. 2013 (6) 
[3] Annette M. Lim, June Corry,Marnie Collins,Lester Peters, Rodney J. Hicks, Ieta D'Costa, Andrew Coleman, Margaret Chua, Benjamin Solomon, Danny Rischin. A phase II study of induction carboplatin and gemcitabine followed by chemoradiotherapy for the treatment of locally advanced nasopharyngeal carcinoma[J]. Oral Oncology. 2013(10)

[4] Wei Lu Kuang,Qin Zhou,Liang Fang Shen. Outcomes and prognostic factors of conformal radiotherapy versus intensity-modulated radiotherapy for nasopharyngeal carcinoma[J]. Clinical and Translational Oncology. 2012 (10)

[5] Sheng-Fa Su, Fei Han, Chong Zhao, Chun-Yan Chen,Wei-Wei Xiao,Jia-Xin Li,Tai-Xiang Lu. Long-Term Outcomes of Early-Stage Nasopharyngeal Carcinoma Patients Treated With Intensity-Modulated Radiotherapy Alone[J]. International Journal of Radiation Oncology, Biology, Physics. 2012 (1)

[6] Ivan Toledano, Pierre Graff, Antoine Serre, Pierre Boisselier, René-Jean Bensadoun, Cécile Ortholan,Pascal Pommier,Séverine Racadot,Gilles Calais,Marc Alfonsi,Veronique Favrel,Philippe Giraud,Michel Lapeyre. Intensity-modulated radiotherapy in head and neck cancer: Results of the prospective study GORTEC 2004-03[J]. Radiotherapy and Oncology. 2012 (1)

[7] Tingting Xu, Chaosu Hu, Guopei Zhu, Xiayun He, Yongru Wu, Hongmei Ying. Preliminary results of a phase III randomized study comparing che motherapy neoadjuvantly or concurrently with radiotherapy for locoregionally advanced nasopharyngeal carcinoma[J]. Medical Oncology. $2012(1)$

[8] Nancy Y Lee,Qiang Zhang,David G Pfister, John Kim,Adam S Garden,James Mechalakos, Kenneth Hu,Quynh T Le,A Dimitrios Colevas,Bonnie S Glisson,Anthony TC Chan, K Kian Ang. Addition of bevacizumab to standard chemoradiation for locoregionally advanced nasopharyngeal carcinoma (RTOG 0615): a phase 2 multi-institutional trial[J]. Lancet Oncology. 2013 (2)

[9] Shu-Zhen Lai, Wen-Fei Li,Lei Chen, Wei Luo, Yuan-Yuan Chen, Li-Zhi Liu, Ying Sun, Ai-Hua Lin, Meng-Zhong Liu, Jun Ma. How Does Intensity-Modulated Radiotherapy Versus Conventional Two-Dimensional Radiotherapy Influence the Treatment Results in Nasopharyngeal Carcinoma Patients[J]. International Journal of Radiation Oncology, Biology, Physics. 2013(3)

[10] Frank C.S. Wong,Alice W.Y. Ng, Victor H.F. Lee, Collin M.M. Lui, Kwok-Keung Yuen, Wing-Kin Sze, To-Wai Leung, Stewart Y. Tung. Whole-Field Simultaneous Integrated-Boost Intensity-Modulated Radiotherapy for Patients With Nasopharyngeal Carcinoma[J]. International Journal of Radiation Oncology, Biology, Physics. 2013 (1) 